\title{
Model-Based Approach to Automated Segmentation of Electron Tomographic Reconstructions.
}

\author{
B. F. McEwen***, M. Jiang***, W. Zhang*, K. Vandenbeldt*, and Qiang Ji*** \\ *Wadsworth Center, New York State Dept Health, Albany, NY \\ **School of Public Health, State University of New York, Albany, NY. \\ ***Rensselaer Polytechnic Institute, Troy, NY
}

Improved instrumentation and methods for automated data collection have made it possible to apply electron tomography to frozen-hydrated specimens, and to obtain high-throughput with plastic-embedded specimens prepared by high-pressure freezing and freeze-substitution [1,2]. High throughput tomography in combination with RNAi disruption of gene expression is a powerful approach for addressing structural-functional questions in cell biology. Currently the most common bottleneck is the limited availability of automated segmentation methods that are effective for tomographic reconstructions of cellular material prepared with minimal extraction of the cytoplasm $[3,4]$. We have addressed this need by developing a model-based approach to segment kinetochore microtubule (kMT) plus-ends from tomographic reconstructions $[5,6]$. kMTs are particularly difficult because their plus-ends are embedded in a complex fibrous mat rich in features with similar gray-scale values (Fig. 1a). Fig. 1b shows a radial slice from a subvolume containing the kMT indicated in Fig 1a. The subvolume was obtained by manually determining two points along the cylindrical axis of the kMT, but we are also refining the fully automated version of this algorithm. The subvolume is enhanced by application of a median filter to remove speckle noise and an anisotropic invariant wavelet filer to enhance elongated structures (Fig 1c). This is followed by a 3D surface-enhancing filer based on eigenvalues of a Weingarten matrix derived from image derivatives and the Hough transform to detect circular contours from the enhanced kMT in cross-sectional view. Centers of the circles are used to calculate the kMT centerlines and false detections are removed by robust fitting to the known geometry. The model is then thinned to a binary image (Fig 1d) and the ends of the kMTs located as the point where detected circles deviate too much from estimated centerlines. The splayed plus-ends of disassembling kMTs are traced in radial slices collected about the centerline of the tubular portion. The tracing is performed in a probabilistic way based on particle filter with higher order prior dynamics to remove features inconsistent with the known curvature of MT protofilaments. A surface-rendered image is shown in Fig 1e. Segmented kMTs can then be placed back into the reconstruction volume and displayed with appropriated 3D imaging software (Figs 2a, 2b). From an axial view it is evident that the kMTs do not overlap one another (Fig 2c), while a higher magnification view shows the kMT plus ends penetrating into the kinetochore. With small modification the method can be used to segment actin and other filamentous structures, and the same principles can be applied to segment tools for membranes, spherical vesicles, and other major cellular substructures in an efficient and unbiased manner.

\section{References}

[1] A.J. Koster et al., J. Struct. Biol. 120 (1997), 276.

[2] K. Vandenbeldt et al., Microsc. Microanal. 10 (Suppl. 2) (2004), 1194CD.

[3] B.F. McEwen and M. Marko, J. Histochem. Cytochem. 49 (2001) 553. 
[4] R. McIntosh et. al., Trends Cell Biol. 15 (2005) 43.

[5] M. Jiang et al., IEEE Eng. Med. Biol. 26 (2004) CD.

[6] M. Jiang et al., IEEE Work App. Comp. Vision (2005) 336.

[7] Supported by NIH grants GM066270, RR01219, and NSF grant DMS0241182.
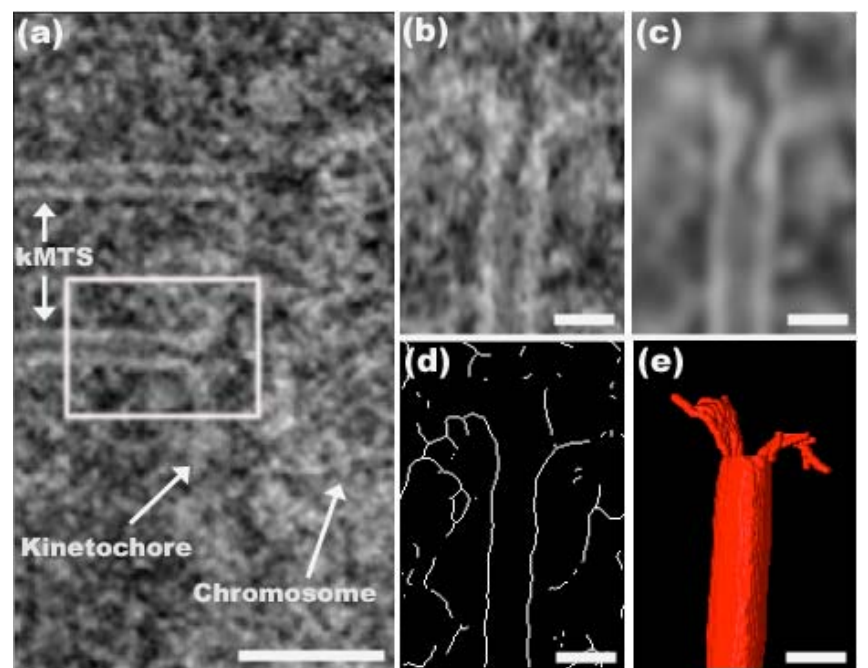

Fig. 1. Model-based segmentation. (a) A single $1.6 \mathrm{~nm}$ thick xy slice from the tomographic reconstruction of a kinetochore in a $\mathrm{PtK}_{1}$ cell prepared by high-pressure freezing and freezesubstitution. The kinetochore and two kMTs are indicated. (b) Radial slice through a subvolume containing the kMT indicated by the box in (a). (c) Enhance version of (b) (see text). (d) Thinned, binary version of the same image after surface enhancement (see text). (e) Surfacerendered version of (d) after tracing guided by model-based predictions. Scale bars $=100 \mathrm{~nm}$ in (a), $25 \mathrm{~nm}$ in (b)-(e).
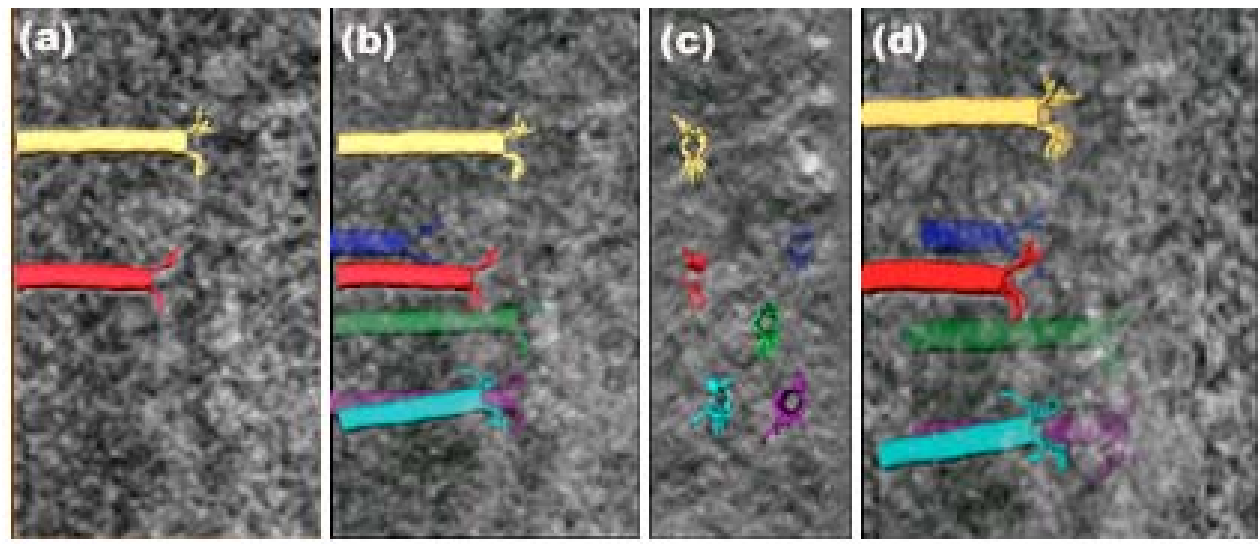

Fig 2. Tomographic reconstruction displayed with six segmented kMTs using Amira visualization software. (a) Same xy slice as shown in 1(a) with two segmented kMTs visible. (b) A view through the volume (transparency turned on) from the xy direction. All of the segmented kMTs are visible. (c) A zy view showing the kMT plus ends in relation to one another. (d) A higher magnification view off the major volume axes showing a variable penetration of kMTs into the kinetochore. 